Vietnam Journal of Mechanics, VAST, Vol.37, No.3 (2015), pp. $217-230$

DOI:10.15625/0866-7136/37/3/6474

\title{
EXTENSION OF DUAL EQUIVALENT LINEARIZATION TECHNIQUE TO FLUTTER ANALYSIS OF TWO DIMENSIONAL NONLINEAR AIRFOILS
}

\author{
Nguyen Minh Triet \\ VNU University of Engineering and Technology, Hanoi, Vietnam \\ *E-mail: 4triet@gmail.com \\ Received June 26, 2015
}

\begin{abstract}
This paper extends the dual equivalent linearization technique (ELT) to obtain flutter speeds of a two-dimensional airfoil with nonlinear stiffness and damping in pitch degree of freedom. Although the use of dual ELT has been investigated in some previous papers, this paper presents an extension of dual ELT using the global-local approach, in which the local equivalent linearization coefficients are averaged in the global sense. The numerical calculation shows that the extended dual ELT gives more accurate flutter speeds in comparison with the ones of classical ELT.

Keywords: Airfoil flutter, dual equivalent linearization technique, global-local approach, limit cycle oscillation, nonlinearity.
\end{abstract}

\section{INTRODUCTION}

The most dramatic physical phenomenon in the field of aeroelasticity is flutter, a dynamic instability which often leads to catastrophic structural failure. Classical theories of linear flutter have been presented for a long time [1,2]. However, the real system exhibits the nonlinear behavior due to the control mechanisms or the connecting parts between wing, pylon, engine, external stores or the large deflection. Nonlinear airfoil flutter is a typical self-excited vibration with rich nonlinear dynamical behaviors, such as limit cycle oscillation (LCO), bifurcation and chaos [3]. Nonlinear aeroelasticity has been a subject of high interest the literature is now extensive $[4,5]$, in which many issues are still under active investigation. In the context of nonlinear aeroelasticity, a LCO is one of the simplest dynamic bifurcations but is a good general description for many nonlinear aeroelastic behaviors. The LCO may occur once the dynamic stability (flutter) boundary has been exceeded. The LCO of the airfoils with stiffness nonlinearities has been investigated by several methods such as harmonic balance method [6,7], center manifold theory [8], point transformation method [9], perturbation-incremental method [10],

(C) 2015 Vietnam Academy of Science and Technology 
precise integration method [11], numerical methods $[12,13]$ and equivalent linearization technique (ELT) [14,15].

The ELT was widely applied to various nonlinear vibration problems because of its simplicity and effectiveness. The approximate solution obtained by the ELT gives some clear physical explanation of the nonlinear phenomenon. The most important procedure of ELT is to derive an equivalent linear system based on some certain equivalent conditions. In the field of dynamical stochastic nonlinear system, the ELT has a long history of development and a numerous versions of ELT have been proposed [16]. Recently, in a series of papers, Anh et al. [17-21] proposed and proved the effectiveness of a so-called dual ELT in nonlinear stochastic systems. The dual approach has been also successfully applied to the problem of tuned mass damper design [21-23]. In [20,23], the globallocal approach applied to the dual criterion has been presented. In this paper, using the global-local approach, we extend the dual ELT by averaging the equivalent linearization coefficients and apply successfully the extended ELT to the flutter LCO problem.

\section{EQUATIONS OF MOTION}

Fig. 1 shows a classical spring-supported airfoil section. The model represents an airfoil with a single bending and a single torsional mode. This two-dimensional model is a typical section of a three-dimensional wing, where the spring constants are adjusted to match the actual uncoupled free vibration frequencies of the wing, while the mass and geometric properties are taken as those of a typical section. The two-dimensional model can give the approximated critical flutter speed of the actual wing with the location of the typical section is generally taken in the neighborhood of 0.7 span from the root [2].

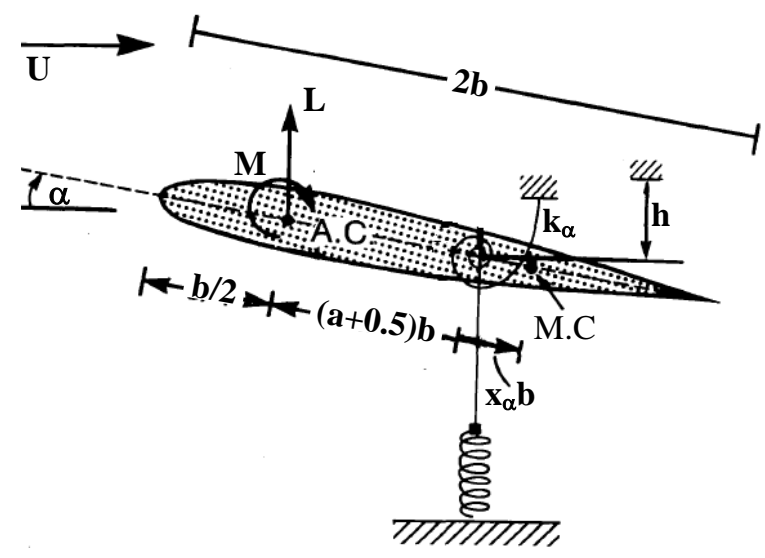

Fig. 1. Airfoil section supported by vertical and torsional springs

As shown in Fig. 1, the airfoil semi-chord is denoted as $b$. The elastic axis of the model is located at a distance $a b$ from the mid-chord. The mass center (M.C) is located at a distance $x_{\alpha} b$ from the elastic axis. The plunge deflection (vertical displacement at the elastic axis) $h$ is positive downward. The pitch angle (angle of twist) $\alpha$ is positive 
nose-up about the elastic axis. The equation of motion of the aeroelastic model has the form [24-28]

$$
\left[\begin{array}{cc}
m_{T} & m_{W} x_{\alpha} b \\
m_{W} x_{\alpha} b & I_{\alpha}
\end{array}\right]\left[\begin{array}{c}
\ddot{h} \\
\ddot{\alpha}
\end{array}\right]+\left[\begin{array}{cc}
c_{h} & 0 \\
0 & c_{\alpha}
\end{array}\right]\left[\begin{array}{c}
\dot{h} \\
\dot{\alpha}
\end{array}\right]+\left[\begin{array}{cc}
k_{h} & 0 \\
0 & k_{\alpha}
\end{array}\right]\left[\begin{array}{l}
h \\
\alpha
\end{array}\right]+\left[\begin{array}{c}
0 \\
g(\alpha, \dot{\alpha})
\end{array}\right]=\left[\begin{array}{c}
-L \\
M
\end{array}\right],
$$

where $m_{w}$ is the mass of the wing, $m_{T}$ is the total mass, $I_{\alpha}$ is mass moment of inertia, $k_{h}$ and $k_{\alpha}$ are the linear plunge and pitch stiffness, $c_{h}$ and $c_{\alpha}$ are the linear damping coefficients, $g(\alpha, \dot{\alpha})$ is a general nonlinear function of the pitch angle $\alpha$ and its derivative $\dot{\alpha}$. The aerodynamic moment $M$ and lift $L$ are assumed having the quasi-steady form

$$
\begin{aligned}
& L=\rho U^{2} b s c_{l \alpha}\left(\alpha+\frac{\dot{h}}{U}+\left(\frac{1}{2}-a\right) b \frac{\dot{\alpha}}{U}\right), \\
& M=\rho U^{2} b^{2} s c_{m \alpha}\left(\alpha+\frac{\dot{h}}{U}+\left(\frac{1}{2}-a\right) b \frac{\dot{\alpha}}{U}\right),
\end{aligned}
$$

where $U$ is the free stream velocity, $\rho$ is the air density, $s$ is the wing section span, $c_{l \alpha}$ and $c_{m \alpha}$ are the aerodynamic lift and moment coefficients per angle of attack. The equation of motion (1) can be transformed to the following state-space form

$$
\left[\begin{array}{c}
\dot{h} \\
\dot{\alpha} \\
\ddot{h} \\
\ddot{\alpha}
\end{array}\right]=\left[\begin{array}{cccc}
0 & 0 & 1 & 0 \\
0 & 0 & 0 & 1 \\
-k_{1} & -k_{2}(U) & -c_{1}(U) & -c_{2}(U) \\
-k_{3} & -k_{4}(U) & -c_{3}(U) & -c_{4}(U)
\end{array}\right]\left[\begin{array}{c}
h \\
\alpha \\
\dot{h} \\
\dot{\alpha}
\end{array}\right]-\left[\begin{array}{c}
0 \\
0 \\
p_{1} \\
p_{2}
\end{array}\right] g(\alpha, \dot{\alpha}),
$$

where the coefficients are defined as

$$
\begin{gathered}
d=m_{T} I_{\alpha}-\left(m_{W} x_{\alpha} b\right)^{2}, k_{1}=\frac{k_{h} I_{\alpha}}{d}, k_{3}=\frac{-m_{W} x_{\alpha} b k_{h}}{d}, p_{1}=\frac{-b m_{W} x_{\alpha}}{d}, p_{2}=\frac{m_{T}}{d}, \\
k_{2}=\frac{\rho U^{2} b s}{d}\left(m_{W} x_{\alpha} b^{2} c_{m \alpha}+I_{\alpha} c_{l \alpha}\right), k_{4}=\frac{-\rho U^{2} b^{2} s}{d}\left(m_{T} c_{m \alpha}+m_{W} x_{\alpha} c_{l \alpha}\right), \\
c_{1}=\frac{\rho b s\left(m_{W} x_{\alpha} b^{2} c_{m \alpha}+c_{l \alpha} I_{\alpha}\right) U+c_{h} I_{\alpha}}{d}, \\
c_{2}=-\frac{\rho U b^{2} s\left(m_{W} x_{\alpha} b^{2} c_{m \alpha}+I_{\alpha} c_{l \alpha}\right)\left(a-\frac{1}{2}\right)+b m_{W} x_{\alpha} c_{\alpha}}{d}, \\
c_{3}=-\frac{\rho U b^{2} s\left(m_{T} c_{m \alpha}+m_{W} x_{\alpha} c_{l \alpha}\right)+b m_{W} x_{\alpha} c_{h}}{d}, \\
c_{4}=\frac{\rho U b^{3} s\left(m_{T} c_{m \alpha}+m_{W} x_{\alpha} c_{l \alpha}\right)\left(a-\frac{1}{2}\right)+m_{T} c_{\alpha}}{d} .
\end{gathered}
$$

\section{EQUIVALENT LINEARIZATION TECHNIQUES}

\subsection{Classical ELT}

Consider the following flutter LCO response

$$
\alpha=A \sin \varphi, \quad \dot{\alpha}=B \cos \varphi,
$$

where $A$ and $B$ respectively are the amplitudes of the pitch angle and its derivative, $\varphi=\omega t$ where $\omega$ is the LCO frequency. The nonlinear function $g$ is linearized as

$$
g(\alpha, \dot{\alpha})=k_{e} \alpha+c_{e} \dot{\alpha}
$$


where the equivalent stiffness $k_{e}$ and equivalent damping $c_{e}$ are found by a certain optimal criterion. There are many criteria for this purpose but the most extensively used criterion is the mean square error criterion which requires the following error be minimum

$$
\int_{0}^{2 \pi}\left(g(\alpha, \dot{\alpha})-k_{e} \alpha-c_{e} \dot{\alpha}\right)^{2} d \varphi \rightarrow \min _{k_{e}, c_{e}}
$$

The minimum conditions

$$
\frac{\partial \int_{0}^{2 \pi}\left(g(\alpha, \dot{\alpha})-k_{e} \alpha-c_{e} \dot{\alpha}\right)^{2} d \varphi}{\partial k_{e}}=0, \quad \frac{\partial \int_{0}^{2 \pi}\left(g(\alpha, \dot{\alpha})-k_{e} \alpha-c_{e} \dot{\alpha}\right)^{2} d \varphi}{\partial c_{e}}=0,
$$

yield

$$
k_{e}=\frac{\int_{0}^{2 \pi} g \alpha d \varphi}{\int_{0}^{2 \pi} \alpha^{2} d \varphi}, \quad c_{e}=\frac{\int_{0}^{2 \pi} g \dot{\alpha} d \varphi}{\int_{0}^{2 \pi} \dot{\alpha}^{2} d \varphi}
$$

using (4) in (7) gives

$$
k_{e}=\frac{1}{\pi A} \int_{0}^{2 \pi} g(A \sin \varphi, B \cos \varphi) \sin \varphi d \varphi, \quad c_{e}=\frac{1}{\pi B} \int_{0}^{2 \pi} g(A \sin \varphi, B \cos \varphi) \cos \varphi d \varphi
$$

for a certain type of nonlinear function, the equivalent stiffness $k_{e}$ and damping $c_{e}$ in (8) are the functions of the pitch amplitude $A$ and pitch velocity amplitude $B$.

\subsection{Dual ELT and its extension}

In [17-20], Anh et al. proposed a so-called dual criterion to obtain a new ELT. This type of ELT has showed its effectiveness in analyzing a numerous class of nonlinear stochastic systems. In [18], the authors stated that the classical ELT is based on replacing the original nonlinear system by an equivalent linear one. The dual approach does not only consider this "forward" replacement but also the "backward" replacement, in which the obtained equivalent linear system is replaced by another nonlinear one that belongs to the same class as the original nonlinear system. In [19], the authors also proposed the weighted dual criterion, which is used in the following dual criterion of this paper

$$
(1-p) \int_{0}^{2 \pi}\left(g(\alpha, \dot{\alpha})-k_{e} \alpha-c_{e} \dot{\alpha}\right)^{2} d \varphi+p \int_{0}^{2 \pi}\left(\lambda g(\alpha, \dot{\alpha})-k_{e} \alpha-c_{e} \dot{\alpha}\right)^{2} d \varphi \rightarrow \min _{k_{e}, c_{e}, \lambda}
$$

where the first term describes the conventional replacement and second term is its dual replacement, $p$ is a given weighting coefficient. If $p=0$ we obtain the classical ELT. If 
$p=1 / 2$ we obtain the dual ELT presented in [18]. Three minimum conditions of (9)

$$
\frac{\partial\left((1-p) \int_{0}^{2 \pi}\left(g(\alpha, \dot{\alpha})-k_{e} \alpha-c_{e} \dot{\alpha}\right)^{2} d \varphi+p \int_{0}^{2 \pi}\left(\lambda g(\alpha, \dot{\alpha})-k_{e} \alpha-c_{e} \dot{\alpha}\right)^{2} d \varphi\right)}{\partial k_{e}}=0,
$$

$\frac{\partial\left((1-p) \int_{0}^{2 \pi}\left(g(\alpha, \dot{\alpha})-k_{e} \alpha-c_{e} \dot{\alpha}\right)^{2} d \varphi+p \int_{0}^{2 \pi}\left(\lambda g(\alpha, \dot{\alpha})-k_{e} \alpha-c_{e} \dot{\alpha}\right)^{2} d \varphi\right)}{\partial c_{e}}=0$,

$\frac{\partial\left((1-p) \int_{0}^{2 \pi}\left(g(\alpha, \dot{\alpha})-k_{e} \alpha-c_{e} \dot{\alpha}\right)^{2} d \varphi+p \int_{0}^{2 \pi}\left(\lambda g(\alpha, \dot{\alpha})-k_{e} \alpha-c_{e} \dot{\alpha}\right)^{2} d \varphi\right)}{\partial \lambda}=0$,

yield

$$
k_{e}=\frac{1-p}{1-p \mu} \frac{\int_{0}^{2 \pi} g \alpha d \varphi}{\int_{0}^{2 \pi} \alpha^{2} d \varphi}, \quad c_{e}=\frac{1-p}{1-p \mu} \frac{\int_{0}^{2 \pi} g \dot{\alpha} d \varphi}{\int_{0}^{2 \pi} \dot{\alpha}^{2} d \varphi}
$$

where $p$ as stated above is a given value between 0 and $1 / 2\left(0 \leq p \leq \frac{1}{2}\right), \mu$ is a notation to simplify the formula and is given by

$$
\mu=\frac{\left(\int_{0}^{2 \pi} g \alpha d \varphi\right)^{2}}{\left(\int_{0}^{2 \pi} g^{2} d \varphi\right)\left(\int_{0}^{2 \pi} \alpha^{2} d \varphi\right)}+\frac{\left(\int_{0}^{2 \pi} g \dot{\alpha} d \varphi\right)^{2}}{\left(\int_{0}^{2 \pi} g^{2} d \varphi\right)\left(\int_{0}^{2 \pi} \dot{\alpha}^{2} d \varphi\right)} .
$$

Using (4) in (13) and (14) gives

$$
\begin{aligned}
& k_{e}=\frac{1-p}{\pi A(1-p \mu)} \int_{0}^{2 \pi} g(A \sin \varphi, B \cos \varphi) \sin \varphi d \varphi, \\
& c_{e}=\frac{1-p}{\pi B(1-p \mu)} \int_{0}^{2 \pi} g(A \sin \varphi, B \cos \varphi) \cos \varphi d \varphi,
\end{aligned}
$$




$$
\mu=\frac{\left(\int_{0}^{2 \pi} g(A \sin \varphi, B \cos \varphi) \sin \varphi d \varphi\right)^{2}+\left(\int_{0}^{2 \pi} g(A \sin \varphi, B \cos \varphi) \cos \varphi d \varphi\right)^{2}}{\pi \int_{0}^{2 \pi}(g(A \sin \varphi, B \cos \varphi))^{2} d \varphi},
$$

However, the equivalent stiffness and damping (15) obtained from the weighted dual criterion ELT (9) depend on the weighting coefficient $p$. In this sense, the equivalent stiffness $k_{e}$ and damping $c_{e}$ in (15) can be considered as local equivalent linearization coefficients. It allows the flexibility in varying the local parameter $p$. However, the main disadvantage of the local solution is that there is still no clear way to find the parameter $p$. Using the global-local approach presented in [20,23], it is suggested that instead of finding a special value of $p$, one may consider its varying in the global domain of integration. Thus, the equivalent coefficients can be suggested as the mean values of all local equivalent coefficients as follows

$$
\begin{aligned}
& k_{e g}=2 \int_{0}^{1 / 2} k_{e} d p=2 \int_{0}^{1 / 2}\left[\frac{1-p}{\pi A(1-p \mu)} \int_{0}^{2 \pi} g(A \sin \varphi, B \cos \varphi) \sin \varphi d \varphi\right] d p, \\
& c_{e g}=2 \int_{0}^{1 / 2} c_{e} d p=2 \int_{0}^{1 / 2}\left[\frac{1-p}{\pi B(1-p \mu)} \int_{0}^{2 \pi} g(A \sin \varphi, B \cos \varphi) \cos \varphi d \varphi\right] d p,
\end{aligned}
$$

in which $k_{e g}$ and $c_{e g}$, respectively, denote the equivalent stiffness and damping obtained by global-local approach. The integrals reduce to

$$
\begin{aligned}
& k_{e g}=\left(\frac{1}{\mu}+\frac{2(1-\mu)}{\mu^{2}} \ln \left(1-\frac{\mu}{2}\right)\right) \frac{\int_{0}^{2 \pi} g(A \sin \varphi, B \cos \varphi) \sin \varphi d \varphi}{\pi A}, \\
& c_{e g}=\left(\frac{1}{\mu}+\frac{2(1-\mu)}{\mu^{2}} \ln \left(1-\frac{\mu}{2}\right)\right) \frac{\int_{0}^{2 \pi} g(A \sin \varphi, B \cos \varphi) \cos \varphi d \varphi}{\pi B} .
\end{aligned}
$$

In brief, the extended dual ELT gives the equivalent stiffness and damping as (18) where the notation $\mu$ is taken from (16).

\section{FLUTTER SPEED PREDICTION AND STABILITY ANALYSIS}

\subsection{Flutter speed prediction}

We will use the equivalent linear coefficients (8) and (18) for further flutter analysis. The linearized equation of (3) has form 


$$
\left[\begin{array}{c}
\dot{h} \\
\dot{\alpha} \\
\ddot{h} \\
\ddot{\alpha}
\end{array}\right]=\left[\begin{array}{cccc}
0 & 0 & 1 & 0 \\
0 & 0 & 0 & 1 \\
-k_{1} & -k_{2}-p_{1} k_{e} & -c_{1} & -c_{2}-p_{1} c_{e} \\
-k_{3} & -k_{4}-p_{2} k_{e} & -c_{3} & -c_{4}-p_{2} c_{e}
\end{array}\right]\left[\begin{array}{c}
h \\
\alpha \\
\dot{h} \\
\dot{\alpha}
\end{array}\right] .
$$

The response of (19) is convergent when the flow velocity is smaller than a critical value (flutter speed). When the flow velocity is higher than flutter speed, the LCO will occur. We can assume the occurrence of following phenomenon. When the flow velocity is smaller than flutter speed, the linear system (19) has two pairs of conjugate poles with negative real parts, which means the convergence of the response. When the flow velocity reaches the flutter speed, a pair of conjugate poles becomes purely imaginary, which means the occurrence of LCO. Denote the poles of (19) at flutter speed as $\pm i \omega,-\zeta_{1} \pm$ $i \omega_{1}$, where $i$ is the imaginary unit, $\omega$ is the LCO frequency as denoted above, $\zeta_{1}, \omega_{1}$ are positive real number. The characteristic polynomial of (19) will has form

$$
\begin{aligned}
P(\lambda) & =\operatorname{det}\left(\left[\begin{array}{cccc}
0 & 0 & 1 & 0 \\
0 & 0 & 0 & 1 \\
-k_{1} & -k_{2}-p_{1} k_{e} & -c_{1} & -c_{2}-p_{1} c_{e} \\
-k_{3} & -k_{4}-p_{2} k_{e} & -c_{3} & -c_{4}-p_{2} c_{e}
\end{array}\right]-\lambda I_{4}\right) \\
& =(\lambda-i \omega)(\lambda+i \omega)\left(\lambda+\zeta_{1}-i \omega_{1}\right)\left(\lambda+\zeta_{1}+i \omega_{1}\right),
\end{aligned}
$$

in which the variable in the brackets are omitted for simplicity, $I_{4}$ is the $4 \times 4$ identity matrix. Equating the coefficients of the polynomials in (20) gives four algebraic equations to solve four variables $\omega, \zeta_{1}, \omega_{1}$ and $U$. The solutions can be obtained in the following form

$$
\begin{gathered}
\zeta_{1}=\frac{c_{1}+c_{4}+p_{2} c_{e}}{2} \\
\omega^{2}=\frac{-c_{3} k_{2}+k_{1}\left(c_{4}+p_{2} c_{e}\right)-k_{3}\left(c_{2}+p_{1} c_{e}\right)+k_{4} c_{1}+p_{2} k_{e} c_{1}-c_{3} p_{1} k_{e}}{c_{1}+c_{4}+p_{2} c_{e}} \\
k_{1} k_{4}-k_{3} k_{2}+k_{1} p_{2} k_{e}-k_{3} p_{1} k_{e}-\omega^{2} p_{2} k_{e}+\omega^{2}\left(c_{2}+p_{1} c_{e}\right) c_{3} \\
-\omega^{2} k_{1}+\omega^{4}-\omega^{2} k_{4}-\omega^{2} c_{1}\left(c_{4}+p_{2} c_{e}\right)=0
\end{gathered}
$$

By substituting $\omega$ from (23) to (24) and by noting that

$$
\omega=\frac{B}{A},
$$

Eqs. (23) and (24) change to

$$
\begin{aligned}
& \frac{B^{2}}{A^{2}}=\frac{-c_{3} k_{2}+k_{1}\left(c_{4}+p_{2} c_{e}\right)-k_{3}\left(c_{2}+p_{1} c_{e}\right)+k_{4} c_{1}+p_{2} k_{e} c_{1}-c_{3} p_{1} k_{e}}{c_{1}+c_{4}+p_{2} c_{e}}, \\
& \left(k_{1}+k_{4}+c_{1} c_{4}-c_{2} c_{3}+p_{2} k_{e}+p_{2} c_{e} c_{1}-p_{1} c_{e} c_{3}\right)\left(c_{1}+c_{4}+p_{2} c_{e}\right) \times \\
& \left(k_{1} c_{4}-c_{3} k_{2}-k_{3} c_{2}+k_{4} c_{1}+p_{2} k_{1} c_{e}+p_{2} k_{e} c_{1}-p_{1} k_{3} c_{e}-p_{1} k_{e} c_{3}\right) \\
& -\left(k_{1} c_{4}-c_{3} k_{2}-k_{3} c_{2}+k_{4} c_{1}+p_{2} k_{1} c_{e}-p_{1} k_{3} c_{e}+p_{2} k_{e} c_{1}-c_{3} p_{1} k_{e}\right)^{2}= \\
& \left(k_{1} k_{4}-k_{3} k_{2}+k_{1} p_{2} k_{\alpha}-k_{3} p_{1} k_{\alpha}\right)\left(c_{1}+c_{4}+p_{2} c_{e}\right)^{2}
\end{aligned}
$$


By noting that the equivalent stiffness $k_{e}$ and equivalent damping $c_{e}$ are the functions of $A$ and $B$ (as seen in (8) or (18)) and $k_{2}, k_{4}, c_{i}(i=1, \ldots, 4)$ are the polynomial functions of flow velocity $U$, Eqs. (25) and (26) give the relation between the flutter speed and the pitch amplitude $A$ or $B$. Moreover, Eq. (26) is a polynomial equation of $U$. After some manipulation, Eq. (26) can reduce to a quintic equation of $U$, whose lowest positive root is the flutter speed.

The relation between $U$ and $A$ can be obtained by the following procedure.

- Given a certain value to $A$.

- By solving the quintic equation (26), we can express $U$ as the function of $B$. It is noted that the quintic equation is solved very fast and efficiently by the "roots" function in MATLAB.

- Substitute $U$ as a function of $B$ to (25), we obtain a nonlinear scalar equation with respect to $B$. It is noted that the nonlinear scalar equation is solved very fast and efficiently by the "fzero" function in MATLAB.

- After solving the nonlinear scalar equation to obtain $B, U$ is determined because it is a function of $B$ as stated above.

- At last, for a certain value of $A$, we can obtain the corresponding value of $U$. Then the relation between $U$ and $A$ can be drawn.

The relation between $U$ and $B$ can be obtained in the similar manner by changing the roles of $A$ and $B$.

\subsection{Stability condition}

For a flutter speed, there can be one or more corresponding pitch amplitude. However, only the stable amplitude can occur in practice. The following condition [29] may be used to determine the stability of LCO in the presence of amplitude perturbations. For a limit cycle with amplitude $A$, the linearized system has two eigenvalues with zero real parts (as shown in (20)). Small perturbations in the limit cycle amplitude make changes in these two eigenvalues. Denote the change of the real part as $\Delta \sigma$. If a positive amplitude perturbation $(\Delta A>0)$ results in the negative real parts $(\Delta \sigma<0)$ of the aforementioned eigenvalues, the LCO is stable because the energy is dissipated until the amplitude decays to its unperturbed value. Similarly, a negative amplitude perturbation $(\triangle A<0)$ results in the positive real parts $(\Delta \sigma>0)$ of the eigenvalues also forms a stable LCO because the amplitude grows until the unperturbed LCO is again attained. In brief, the condition

$$
\Delta \sigma \cdot \Delta A<0
$$

defines a stable LCO.

In calculation, the stability condition is checked by the following procedure. With a certain value of the pitch amplitude $A$, the flutter speed is obtained by the procedure presented in section 3.1. Then the flow velocity is fixed at the flutter speed while the pitch amplitude $A$ is given a small perturbation $\Delta A$. The amplitude of pitch velocity $B$ is obtained by scalar equation ... Then the new roots of the characteristic polynomial $P(\lambda)$ in (20) are obtained. Two purely imaginary roots now become two conjugate roots with nonzero real part, denoted as $\Delta \sigma$. If two perturbations $\Delta \sigma$ and $\Delta A$ satisfy the condition (27), the corresponding state of flutter is stable. 


\section{NUMERICAL SIMULATION}

In this section, two numerical examples presented in [24] are performed to verify the proposed ELTs. In [24], the numerical examples consider the leading-edge and/or trailing-edge control surfaces. However, because the control problem is beyond the scope of this paper, only the open loop systems are analyzed. The nonlinear function $g(\alpha, \dot{\alpha})$ in both examples is expressed in polynomial form up to $5^{\text {th }}$ order as follows

$$
g=k_{\alpha 2} \alpha^{2}+k_{\alpha 3} \alpha^{3}+k_{\alpha 4} \alpha^{4}+k_{\alpha 5} \alpha^{5} .
$$

Substituting the nonlinear form (28) into the formula (16) of $\mu$, and the equivalent stiffnesses (8) or (17) give the following formulas

$$
\begin{gathered}
\mu=\frac{\left(\frac{3 A^{2} k_{\alpha 3}}{4}+\frac{5 A^{4} k_{\alpha 5}}{8}\right)^{2}}{k_{\alpha 5}^{2} \frac{63 A^{8}}{128}+\left(2 k_{\alpha 3} k_{\alpha 5}+k_{\alpha 4}^{2}\right) \frac{35 A^{6}}{64}+\left(2 k_{\alpha 2} k_{\alpha 4}+k_{\alpha 3}^{2}\right) \frac{5 A^{4}}{8}+k_{\alpha 2}^{2} \frac{3 A^{2}}{4}} \\
k_{e}=\gamma\left(\frac{3 A^{2} k_{\alpha 3}}{4}+\frac{5 A^{4} k_{\alpha 5}}{8}\right)
\end{gathered}
$$

where $\gamma$ is the coefficient depending on the ELT used as follows

* For the classical ELT: $\gamma=1$

* For the extended dual ELT: $\gamma=\frac{1}{\mu}+\frac{2(1-\mu)}{\mu^{2}} \ln \left(1-\frac{\mu}{2}\right)$

\subsection{Example 1}

The numerical values of parameters of Example 1 are shown in Tab. 1.

Table 1. Numerical values of parameters of Example 1

\begin{tabular}{|c|c|c|c|c|c|c|}
\hline$A$ & $b(\mathrm{~m})$ & $m_{T}(\mathrm{~kg})$ & $m_{W}(\mathrm{~kg})$ & $I_{\alpha}\left(\mathrm{kg} \cdot \mathrm{m}^{2}\right)$ & $x_{\alpha}$ & $k_{h}(\mathrm{~N} / \mathrm{m})$ \\
\hline-0.6847 & 0.135 & 12.387 & 2.049 & 0.0558 & 0.3314 & 2884.4 \\
\hline$c_{h}(\mathrm{~kg} / \mathrm{s})$ & $c_{l \alpha}$ & $c_{m \alpha}$ & $s(\mathrm{~m})$ & $\rho\left(\mathrm{kg} / \mathrm{m}^{3}\right)$ & $c_{\alpha}\left(\mathrm{kg} \cdot \mathrm{m}^{2} / \mathrm{s}\right)$ & $k_{\alpha}(\mathrm{N} . \mathrm{m})$ \\
\hline 27.43 & 6.38 & -1.16 & 0.6 & 1.225 & 0.036 & 6.833 \\
\hline$k_{\alpha 2}$ (N.m) & $k_{\alpha 3}$ (N.m) & $k_{\alpha 4}$ (N.m) & $k_{\alpha 5}$ (N.m) & & & \\
\hline 9.967 & 667.685 & 26.569 & -5087.931 & & & \\
\hline
\end{tabular}

The numerical solutions are obtained by the ode45 function in MATLAB. The following process is carried out to plot the numerical relation between the flutter speed and the pitch amplitude:

- First, an initial condition of pitch angle $\alpha$ is set. Other initial conditions $(h(0)$, $\dot{h}(0), \dot{\alpha}(0))$ are set to zeros.

- Gradually increase the flow velocity $U$. For each flow velocity, the nonlinear differential equations (19) are solved from 0s to 120s. If the response converges, the flow 
velocity is smaller than the flutter speed. When the response starts to make a LCO, the flow velocity is the flutter speed and the amplitude of LCO is recorded.

- Increase the initial pitch angle $\alpha(0)$ and repeat the step 2. The initial pitch angle is increased until the pitch amplitude has no significant changes.

The relation between the initial pitch angle and the flutter pitch amplitude (calculated numerically) is shown in Fig. 2. Fig. 3 shows the flutter boundary obtained by numerical calculations and two ELTs. Tab. 2 shows the comparisons of some flutter speeds.

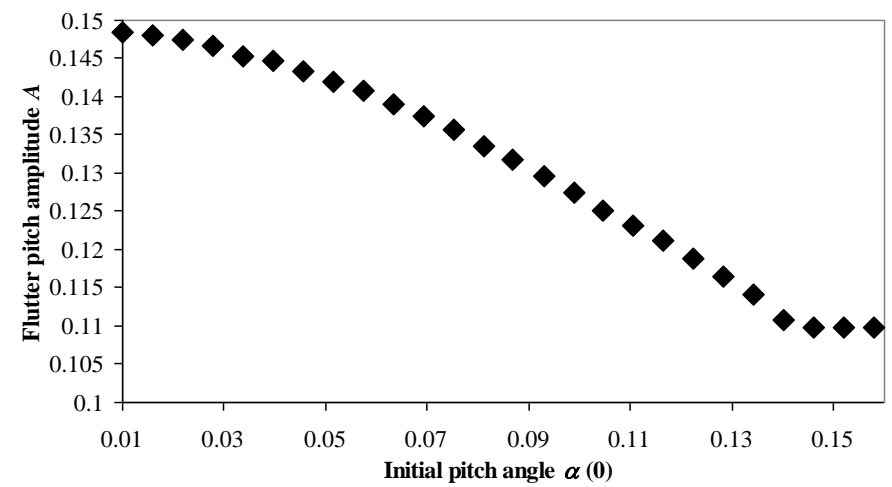

Fig. 2. Relation between initial condition and flutter pitch amplitude in Example 1

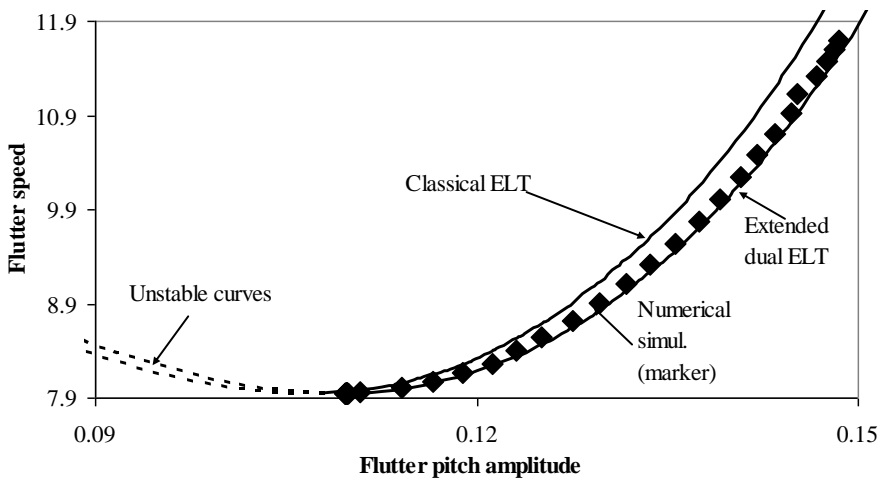

Fig. 3. Relation between flutter speed and flutter pitch amplitude in Example 1

\subsection{Example 2}

The numerical values of parameters of Example 2 are shown in Tab. 3.

The relation between the initial pitch angle and the flutter pitch amplitude (calculated numerically) is shown in Fig. 4 . Fig. 5 shows the flutter boundary obtained by numerical calculations and two ELTs. Tab. 4 shows the comparisons of some flutter speeds. 
Table 2. Comparisons of flutter speeds in Example 1

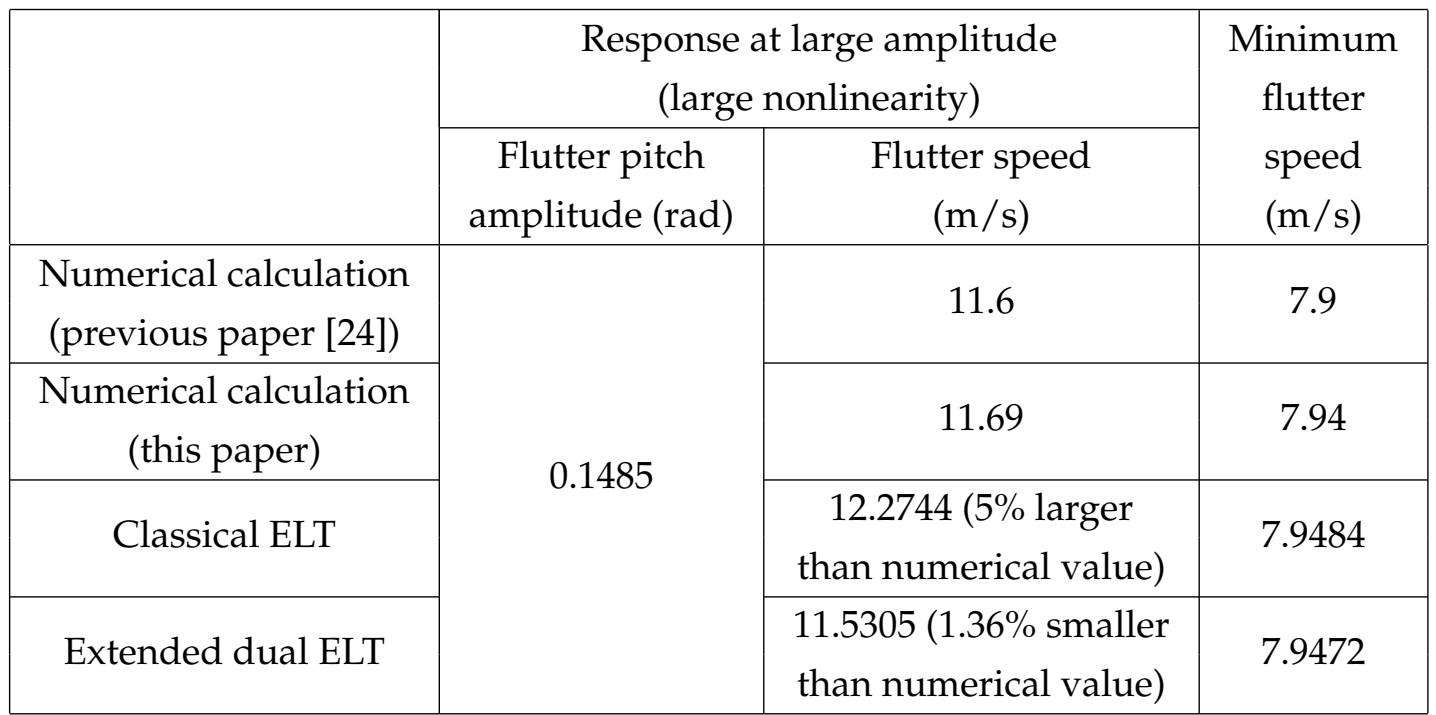

Table 3. Numerical values of parameters of Example 2

\begin{tabular}{|c|c|c|c|c|c|c|}
\hline$A$ & $b(\mathrm{~m})$ & $m_{T}(\mathrm{~kg})$ & $m_{W}(\mathrm{~kg})$ & $I_{\alpha}\left(\mathrm{kg} \cdot \mathrm{m}^{2}\right)$ & $x_{\alpha}$ & $k_{h}(\mathrm{~N} / \mathrm{m})$ \\
\hline-0.6719 & 0.1905 & 15.57 & 5.23 & 0.1419 & 0.5721 & 2844 \\
\hline$c_{h}(\mathrm{~kg} / \mathrm{s})$ & $c_{l \alpha}$ & $c_{m \alpha}$ & $s(\mathrm{~m})$ & $\rho\left(\mathrm{kg} / \mathrm{m}^{3}\right)$ & $c_{\alpha}\left(\mathrm{kg} \cdot \mathrm{m}^{2} / \mathrm{s}\right)$ & $k_{\alpha}(\mathrm{N} . \mathrm{m})$ \\
\hline 27.43 & 6.757 & -1.162 & 0.5945 & 1.225 & 0.0184 & 12.77 \\
\hline$k_{\alpha 2}$ (N.m) & $k_{\alpha 3}$ (N.m) & $k_{\alpha 4}$ (N.m) & $k_{\alpha 5}$ (N.m) & & & \\
\hline 53.47 & 1003 & 0 & 0 & & & \\
\hline
\end{tabular}

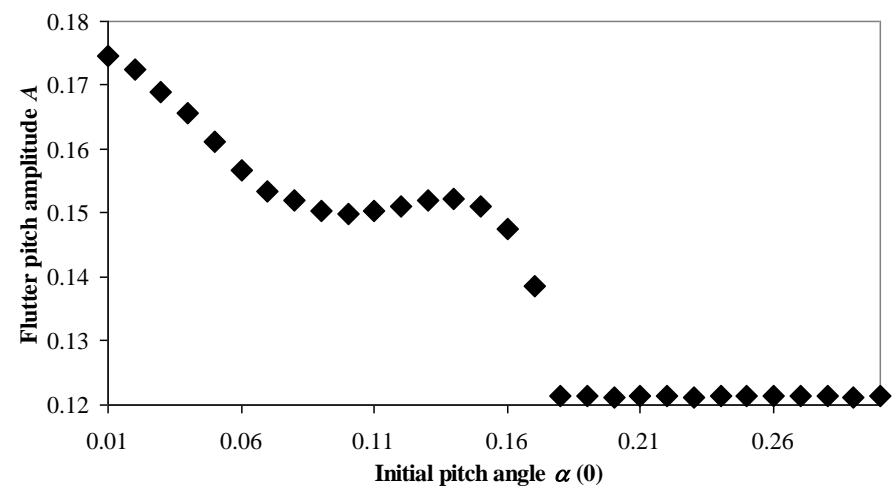

Fig. 4. Relation between initial condition and flutter pitch amplitude in Example 2 


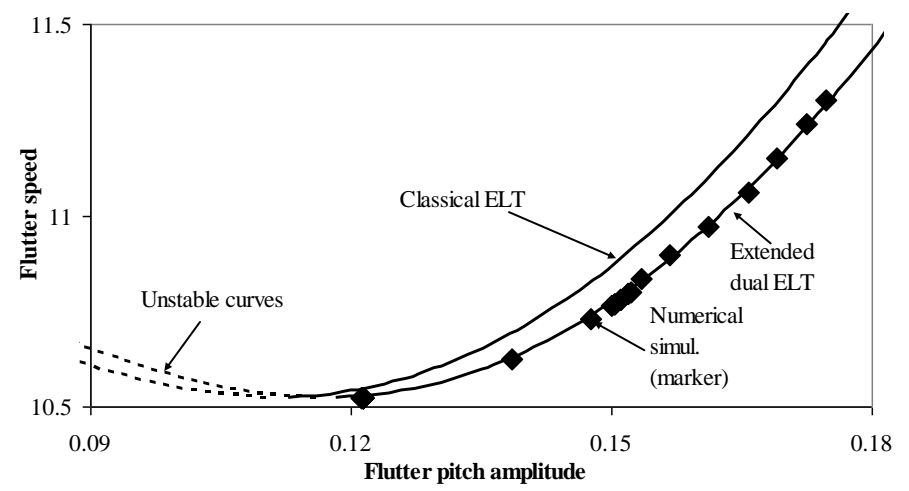

Fig. 5. Relation between flutter speed and flutter pitch amplitude in Example 2

Table 4. Comparisons of flutter speeds in Example 2

\begin{tabular}{|c|c|c|c|}
\hline & \multicolumn{2}{|c|}{$\begin{array}{l}\text { Response at large amplitude } \\
\text { (large nonlinearity) }\end{array}$} & \multirow{2}{*}{$\begin{array}{c}\text { Minimum } \\
\text { flutter } \\
\text { speed } \\
(\mathrm{m} / \mathrm{s})\end{array}$} \\
\hline & $\begin{array}{c}\text { Flutter pitch } \\
\text { amplitude (rad) }\end{array}$ & $\begin{array}{l}\text { Flutter speed } \\
\qquad(\mathrm{m} / \mathrm{s})\end{array}$ & \\
\hline $\begin{array}{l}\text { Numerical calculation } \\
\text { (previous paper [24]) }\end{array}$ & \multirow{4}{*}{0.1746} & 11.4 & 10.6 \\
\hline $\begin{array}{l}\text { Numerical calculation } \\
\text { (this paper) }\end{array}$ & & 11.3 & 10.525 \\
\hline Classical ELT & & $\begin{array}{l}11.4481(1.31 \% \text { larger } \\
\text { than numerical value })\end{array}$ & 10.5249 \\
\hline Extended dual ELT & & $\begin{array}{l}11.2852(0.13 \% \text { smaller } \\
\text { than numerical value })\end{array}$ & 10.5248 \\
\hline
\end{tabular}

\subsection{Discussions on results}

- As seen in Tab. 2 and Tab. 4, the numerical results in this paper are in good agreement with those in previous paper [24]. This guarantees the reliability of the numerical procedure in this paper.

- In Figs. 2 and 4, there is a jump phenomenon in the relation between the initial pitch angle and flutter pitch amplitude. When the initial angle is small, the flutter pitch amplitude suddenly jumps to a large value. Increase the initial angle more, the flutter pitch amplitude decreases and tends to a constant value. The jump phenomenon clearly shows the nonlinear effect.

- In Figs. 3 and 5, all the methods show the existence of a minimum flutter speed. The comparisons in the last column of Tabs. 2 and 4 show that both ELTs can predict the 
minimum flutter speed quite well. It is noted that the ELTs can find the minimum flutter speed quite simply by solving the quintic equation (24).

- The proposed extended dual ELT based on weighted dual criterion and globallocal approach shows its accuracy in the region of large nonlinearity (large amplitude). As shown in Tabs. 3 and 5, in Examples 1 and 2, the errors of the extended dual ELT are about $1 / 3$ and $1 / 10$ of the error of the classical ELT, respectively.

\section{CONCLUSIONS}

The equivalent linearization technique (ELT) in this paper is improved by a socalled weighted dual criterion and global-local approach. The dual criterion consists of "forward" and "backward" replacements with a local weighting coefficient. The obtained local equivalent coefficients are then averaged to be the global one. The proposed ELT then is applied to predict the flutter limit cycle oscillation of an airfoil section with nonlinear pitch stiffness and nonlinear pitch damping. Two numerical examples of the nonlinear polynomial pitch stiffness have been carried out. The results show that the proposed ELT can reduce the error to about 1/3 (in Example 1) and 1/10 (in Example 2) of the error given by the classical ELT. It is expected that the extended dual ELT can be used as an alternative effective tool for flutter analysis of more complicate nonlinear systems.

\section{REFERENCES}

[1] R. D. Blevins. Flow-induced vibration. Van Nostrand Reinhold, New York, 2nd edition, (1990).

[2] Y. C. Fung. An introduction to the theory of aeroelasticity. Dover Edition, (1993).

[3] B. H. K. Lee, S. J. Price, and Y. S. Wong. Nonlinear aeroelastic analysis of airfoils: bifurcation and chaos. Progress in Aerospace Sciences, 35, (3), (1999), pp. 205-334.

[4] E. H. Dowell, J. W. Edwards, and T. Strganac. Nonlinear aeroelasticity. J Aircr, 40, (5), (2003), pp. 857-874.

[5] J. Xiang, Y. Yan, and D. Li. Recent advance in nonlinear aeroelastic analysis and control of the aircraft. Chinese Journal of Aeronautics, 27, (1), (2014), pp. 12-22.

[6] L. Liu and E. H. Dowell. Harmonic balance approach for an airfoil with a freeplay control surface. AIAA Journal, 43, (4), (2005), pp. 802-815.

[7] Y. M. Chen and J. K. Liu. Elliptic harmonic balance method for two degree-of-freedom self-excited oscillators. Communications in Nonlinear Science and Numerical Simulation, 14, (3), (2009), pp. 916-922.

[8] L. Liu, Y. S. Wong, and B. H. K. Lee. Application of the centre manifold theory in non-linear aeroelasticity. Journal of Sound and Vibration, 234, (4), (2000), pp. 641-659.

[9] L. Liu, Y. S. Wong, and B. H. K. Lee. Non-linear aeroelastic analysis using the point transformation method, part 1: Freeplay model. Journal of Sound and Vibration, 253, (2), (2002), pp. 447-469.

[10] K. W. Chung, Y. B. He, and B. H. K. Lee. Bifurcation analysis of a two-degree-of-freedom aeroelastic system with hysteresis structural nonlinearity by a perturbation-incremental method. Journal of Sound and Vibration, 320, (1), (2009), pp. 163-183.

[11] C. C. Cui, J. K. Liu, and Y. M. Chen. Simulating nonlinear aeroelastic responses of an airfoil with freeplay based on precise integration method. Communications in Nonlinear Science and Numerical Simulation, 22, (1), (2015), pp. 933-942. 
[12] R. Vasconcellos and A. Abdelkefi. Nonlinear dynamical analysis of an aeroelastic system with multi-segmented moment in the pitch degree-of-freedom. Communications in Nonlinear Science and Numerical Simulation, 20, (1), (2015), pp. 324-334.

[13] R. Vasconcellos, A. Abdelkefi, M. R. Hajj, and F. D. Marques. Grazing bifurcation in aeroelastic systems with freeplay nonlinearity. Communications in Nonlinear Science and Numerical Simulation, 19, (5), (2014), pp. 1611-1625.

[14] F. X. Chen, Y. M. Chen, and J. K. Liu. Equivalent linearization method for the flutter system of an airfoil with multiple nonlinearities. Communications in Nonlinear Science and Numerical Simulation, 17, (12), (2012), pp. 4529-4535.

[15] F. Chen, J. Liu, and Y. Chen. Flutter analysis of an airfoil with nonlinear damping using equivalent linearization. Chinese Journal of Aeronautics, 27, (1), (2014), pp. 59-64.

[16] L. Socha. Methods for stochastic dynamic systems, Vol. 730. Lecture Notes in Physics, Springer, Berlin Heidelberg, (2008).

[17] N. D. Anh. Duality in the analysis of responses to nonlinear systems. Vietnam Journal of Mechanics, 32, (4), (2010), pp. 263-266.

[18] N. D. Anh, N. N. Hieu, and N. N. Linh. A dual criterion of equivalent linearization method for nonlinear systems subjected to random excitation. Acta Mechanica, 223, (3), (2012), pp. 645-654.

[19] N. D. Anh, V. L. Zakovorotny, N. N. Hieu, and D. V. Diep. A dual criterion of stochastic linearization method for multi-degree-of-freedom systems subjected to random excitation. Acta Mechanica, 223, (12), (2012), pp. 2667-2684.

[20] N. D. Anh, L. X. Hung, and L. D. Viet. Dual approach to local mean square error criterion for stochastic equivalent linearization. Acta Mechanica, 224, (2), (2013), pp. 241-253.

[21] N. D. Anh and N. X. Nguyen. Design of dynamic vibration absorber for damped linear structures. In Proceedings of the Institution of Mechanical Engineers, Part C: Journal of Mechanical Engineering Science, Vol. 228, (2014), pp. 45-55.

[22] N. D. Anh, N. X. Nguyen, and L. T. Hoa. Design of three-element dynamic vibration absorber for damped linear structures. Journal of Sound and Vibration, 332, (19), (2013), pp. 4482-4495.

[23] N. D. Anh, N. X. Nguyen, and N. H. Quan. Global-local approach to the design of dynamic vibration absorber for damped structures. Journal of Vibration and Control, (2014). DOI: $10.1177 / 1077546314561282$.

[24] D. Li, J. Xiang, and S. Guo. Adaptive control of a nonlinear aeroelastic system. Aerospace Science and Technology, 15, (5), (2011), pp. 343-352.

[25] T. W. Strganac, J. Ko, and D. E. Thompson. Identification and control of limit cycle oscillations in aeroelastic systems. Journal of Guidance, Control, and Dynamics, 23, (6), (2000), pp. 11271133.

[26] G. Platanitis and T. W. Strganac. Control of a nonlinear wing section using leading-and trailing-edge surfaces. Journal of Guidance, Control, and Dynamics, 27, (1), (2004), pp. 52-58.

[27] J. Ko, T. W. Strganac, J. L. Junkins, M. R. Akella, and A. J. Kurdila. Structured model reference adaptive control for a wing section with structural nonlinearity. Journal of Vibration and Control, 8, (5), (2002), pp. 553-573.

[28] A. Abdelkefi, R. Vasconcellos, F. D. Marques, and M. R. Hajj. Modeling and identification of freeplay nonlinearity. Journal of Sound and Vibration, 331, (8), (2012), pp. 1898-1907.

[29] X. Wei and J. E. Mottershead. Aeroelastic systems with softening nonlinearity. AIAA Journal, 52, (9), (2014), pp. 1915-1927. 